\title{
Neural modeling of plant tissue cultures: a review
}

\author{
SYLWIA ZIELIŃSKA*, EWA KĘPCZYŃSKA \\ Department of Plant Biotechnology, Faculty of Biology, University of Szczecin, Szczecin, Poland \\ *Corresponding author: sziel@univ.szczecin.pl
}

\begin{abstract}
Artificial neural networks (ANNs) are widely used in science and technology, and have been successfully applied in plant tissue cultures. First of all, ANNs can simulate the growth of plants under different in vitro conditions. Their usefulness has been confirmed in the estimation of biomass in plant cell cultures and the length of shoots in vitro, in the classification of somatic embryos, evaluation of the physical conditions of an in vitro environment, and in the prediction of optimal conditions for in vitro culture to achieve maximum efficiency and productivity. Secondly, with the help of various types of neural models, in vitro-regenerated plants are sorted, respectively, to their quality and likeliness of further development. Thirdly, ANNs are capable of predicting plant behavior during in vitro rhizogenesis and subsequent acclimatization to ex vitroconditions. Several neural and neurofuzzy models for the aforementioned biological processes are reviewed in this paper. In addition, the fundamentals of neural modeling, namely the construction of ANNs, are presented and their flexibility and attractiveness are highlighted.
\end{abstract}

Key words: biomass estimation, embryo classification, neural modeling, plant clustering, prediction of conditions, tissue culture

\section{Introduction}

The in vitro method used for growing plants is one of the most popular methods employed in plant biotechnology. Without in vitro techniques, plant micropropagation, androgenesis, gynogenesis, somatic embryogenesis, or the production of secondary metabolites would not be possible. Such processes can be studied because they take place in controlled conditions like strict sterility, defined temperature, light and humidity as well as on solid or liquid media of a scheduled composition. Although there are many biological processes that can easily be observed in plant tissue cultures, none of them are linear, and, moreover, they are influenced by many other factors as well. Thus, appropriate modeling can be applied to quite accurately predict and simulate the growth kinetics of the culture and also predict the resulting biomass. Conventional analytical techniques based on mathematical models are not suitable for these purposes, because they are not developed for plant in vitro cultures (Prasad and Dutta Gupta, 2008a).

Artificial neural network technology is an effective alternative used for a reliable and objective assessment of biological processes. ANNs, by drawing inspiration from the construction and functioning of the human brain, simulate the brain's key features, such as its ability to learn and to generalize acquired knowledge. These functions are the advantages for creating semantic models of biological processes (Kosiński, 2002). Although ANNs have shown significant progress in controlling bioprocesses, their use in the complex systems of plant tissue cultures is relatively infrequent.

The purpose of this review is to present the techniques of neural modeling as tools for data analysis and their application in plant in vitro cultures for classification, clustering, estimation, prediction and simulation.

\section{Neural modeling basics}

Neural modeling is the creation and modification of any process models with the use of neural networks and implemented in specialized computer programs. The main purpose of modeling is to simplify a complex reality, including the relationships and processes that take place within it. ANNs imitate the construction of models of neural structures in the brain and copy the phenomena that occur in the human nervous system. The basic elements of ANNs are combined together and grouped in a layer of processing elements called neurons. The working of the neural network depends on the number of 


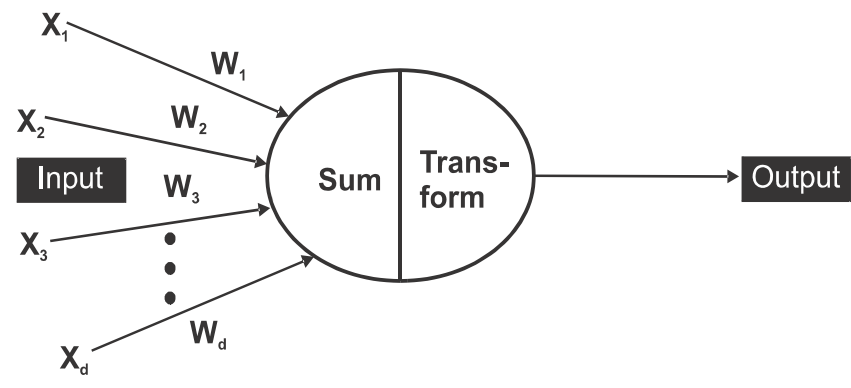

Fig. 1. Structure of single artificial neuron (according to Zhang, 2008)

neurons, the neuron model and the network's architecture. An artificial neuron is composed of input parameters (weights associated with inputs and bias), the postsynaptic potential function, the transfer (activation) function and the output. The proper working of a network requires a proper choice of parameter values, e.g. neuron weights and bias (Tadeusiewicz, 1993; Tadeusiewicz et al., 2007). Inputs are used to enter information into the neuron. Exceeding the bias value activates the neuron. Each neuron processes information in two steps: first, the inputs $\left(\mathrm{x}_{\mathrm{i}}\right)$ are combined together to form a weighted sum of inputs and the weights $\left(\mathrm{w}_{\mathrm{i}}\right)$ of the connecting links (Fig. 1); and then, the neuron converts the sum to an output via a transfer function. The non-linear sigmoid transfer function (Prasad and Dutta Gupta, 2008a) is the most commonly used function in biological systems. In neural modeling, the number of layers and the number and types of neurons in each layer must first be selected. Then, the weights of all neurons are determined in the training process in order to minimize the error of the network. This assignment is achieved by a suitable training algorithm which depends on the type of input data, and should be able to calculate the desired output values. The data from plant in vitro cultures can be classified as: i) binary data which take only two values, such as yes/no, organogenic/non-organogenic; ii) discrete data which consist of more than two values, e.g. the number of proliferated shoots; iii) continuous data which can reach any value, such as callus weight; iv) temporal data; v) time series data; vi) categorical data, such as the type of growth regulator, or the type of response; and vii) fuzzy data that refer to the developmental stages of embryos, callus colour, and degree of vitrification (Prasad and Dutta Gupta, 2008a; Gago et al., 2010c; Gallego et al., 2011). There are many types of ANNs depending on the network topology, the manner in which information flows between the layers, and the construction and connection of single neurons. These ANN types are: i) feedforward networks, e.g. perceptrons, single- and multilayered (MLP); ii) recurrence networks with feedback loops; iii) self-organizing networks, such as Kohonen networks; iv) radial basis function networks (RBFN); v) resonance (ART - adaptive resonance theory); vi) probabilistic and hybrid (fuzzy and neurofuzzy logic) networks.

The most common network architecture used to estimate biological processes is a multilayer perceptron. The MLP consists of three basic layers of neurons: input, hidden and output. The neurons are each connected to all of the units in the preceding layer (Fig. 2). A weighting factor is assigned to each connection. Hidden neurons allow the virtual modeling of any problem and the number of layers of these neurons can be arbitrary (Osowski, 2006). MLP networks are trained by using the "learning with teacher" strategy, i.e. on the example of a set of training data, input data and corresponding output data. Based on these data, the training algorithm modifies the network parameters and produces results as close as possible to the output data. There are many MLP network training algorithms, among which the most frequently chosen are: the backpropagation algorithm, gradient descent, conjugate gradient method, and quasiNewton (variable metric). The application of neural technology requires preparation of a training data set in the training process. The process of training can be controlled by selecting the number of epochs (iterations) for training and the testing of weights on the data from the validation set which is used to assess the quality and capacity of the network to generalize the acquired knowledge. The final assessment of the quality of the network is made after the training comes to an end by using a set of test data based on global error (GE), which is calculated for all test patterns from the set. Quality standards are the neural model error values, such as the sum of the squares of the error (SSE), mean error (ME), root mean square (RMS), mean square error (MSE), and the linear Pearson's correlation coefficient (R). Neural modeling can interfere with the training data set and the network structure to obtain the best-suited model. In most cases, good results can be achieved by using a simple MLP network structure. In order to optimize MLP, changes are made in the number of hidden layers in the network, the number of neurons in these layers, 


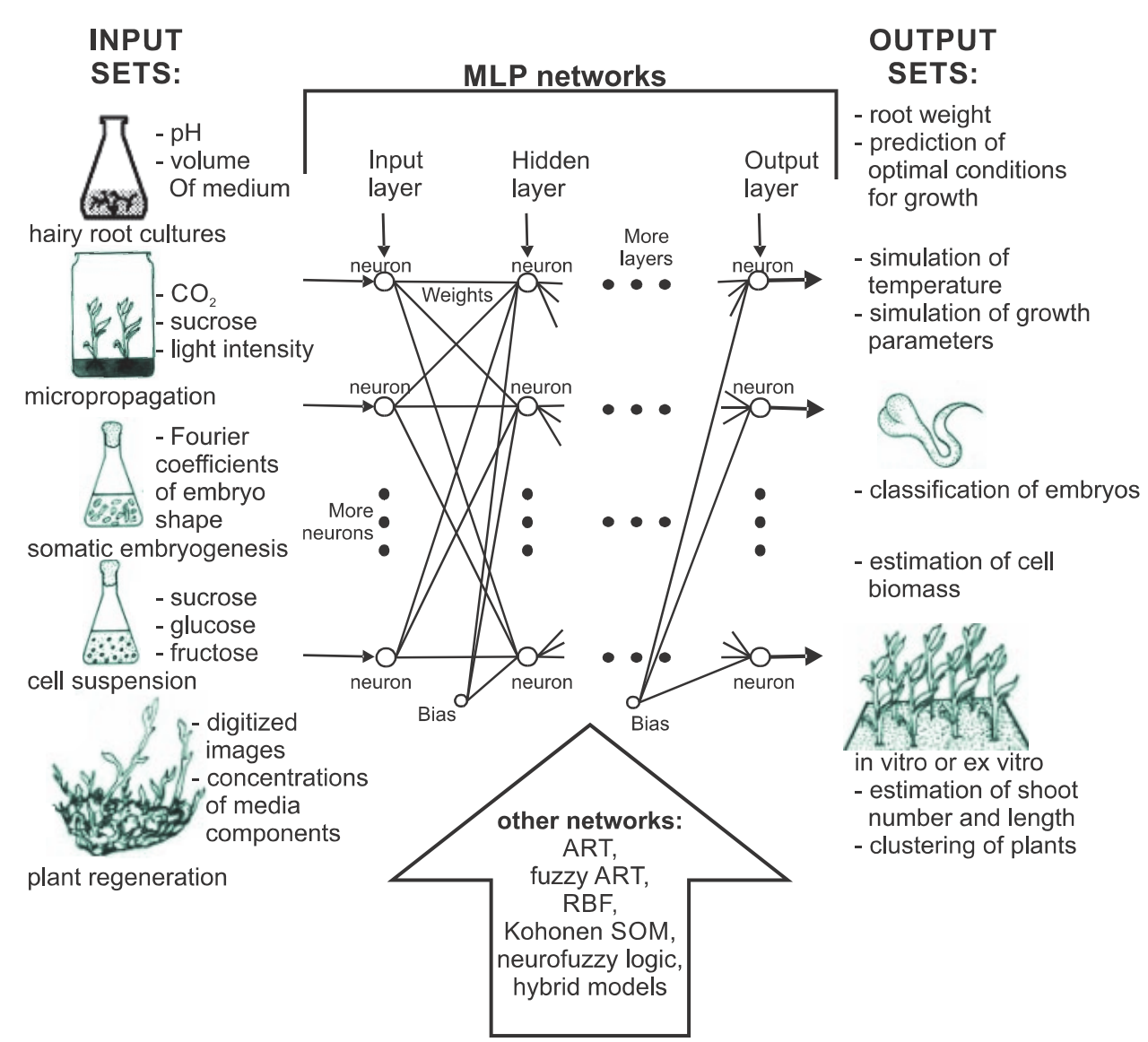

Fig. 2. Structure of MLP networks and actual ANN application in plant tissue cultures (see inputs and outputs)

in the initial weights, type of learning algorithm, momentum, etc., so as to obtain a better prediction of the model during the testing phase (Prasad and Dutta Gupta, 2008a; Sadat Noori et al., 2011).

A frequently chosen network for modeling is RNN - a radial neural network (radial basis function network - RBFN or RBF) with a characteristic topology and structure of neurons in the hidden layer. This network's structure is feedforward and has three layers (Fig. 3). In the input layer, there is one neuron for each predictor variable. In the case of categorical variables, N-1 neurons are used: where $\mathrm{N}$ is the number of categories. The input neurons standardize the range of the values by subtracting the median and dividing by the interquartile range, and then they feed the values to each of the neurons in the hidden layer. The hidden layer has a variable number of neurons (the optimal number is determined by the training process). Each neuron consists of a radial basis function centered on a point with as many dimensions as there are predictor variables (Sherrod, 2013). The spread (radius) of the RBF function may be different for each dimension. The centers and spreads are determined by the training process. When presented with the $\mathrm{x}$ vector of input values from the input layer, a hidden neuron computes the Euclidean distance of the test case from the neuron's center point and then applies the RBF kernel function to this distance using the spread values. The resulting value is passed to the summation layer. The summation layer consists of linear neurons whose number depends on the number of network output parameters. The value proceeding from a neuron in the hidden layer is multiplied by a weight associated with the neuron (W1, W2, Wn in Fig. 3) and passed to the summation, which adds up the weighted values and presents this sum as the output of the network. Not shown in this figure is a bias value of 1.0 that is multiplied by a weight W0 and fed into the summation layer. Due to classification problems, there is one output (and a separate set of weights and summation unit) for each target category. The value output for a category is the probability that the case being evaluated has that category (Tadeusiewicz, 1993; Tadeusiewicz et al., 2007; Sherrod, 2013). 


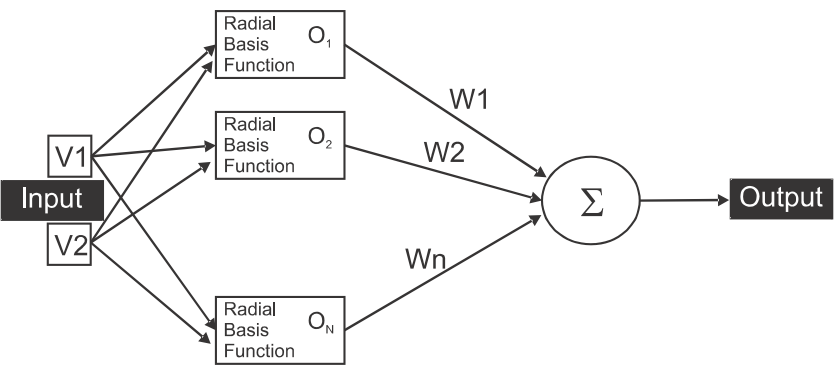

Fig. 3. RBF network architecture (according to Sherrod, 2013)

Another theory widely used in neural modeling is adaptive resonance theory (ART). The performance of resonance networks is associated with ART as developed by G.A. Carpenter and S. Grossberg (1987, by Prasad and Dutta Gupta, 2008a). The theory of ART consists of several concepts (ART1, ART2, ART-2A, ART3) which describe how information is processed by the brain. In these models, it is assumed that both the supervised and the unsupervised manner of information flow will be used to analyze and recognize the patterns - reference data, shapes, sizes. ART1 is a basic model that is used to identify binary signals, and its modification is ART2, which is used for the analysis of continuous data, such as analog signals. The ART-2A model is a simplified continuation of ART2 and enables a much faster calculation. Its development is ART3, which includes an analysis of complex models that occur at the joints of individual neurons, i.e. it simulates the action of a neurotransmitter. In ART systems it is possible to work on continuous data and to analyze them in a fuzzy way - in which there are no clear boundaries between the analyzed individuals. Fuzzy ART neural networks are characterized by intermediate values between the integer values ( 0 and 1 - typical for images). The application of a fuzzy ART network significantly reduces the number of mistakes and errors made during the analysis at every level of diagnosis, analysis and data classification. These networks are flexible and can change their structure with respect to the flow of input signals. Furthermore, they are characterized by two systems of memory (long- and short-lived), corresponding to the human memory. ART models are characterized by a much higher efficiency of training in a shorter period of time compared to training in multilayer networks with backpropagation error. This is because the system remembers the already learnt patterns (Osowski, 2006). Resonance networks consist of two

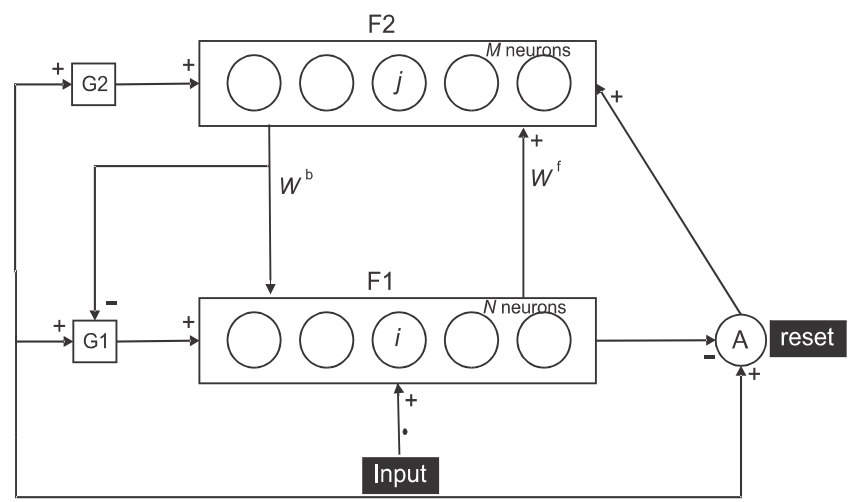

Fig. 4. The ART neural network: F1 - the comparison (bottom) layer, F2 - the recognition (top) layer, G1 and G2 - subsystems of control system, A - orienting system (according to http://www.learnartificialneuralnetworks.com/art.html)

main layers, one at the bottom and one at the top, and also include two systems - control and orienting (Fig. 4). The input shape is stored in the input layer. The other layer, i.e. the output (top), is responsible for identifying the class to which the input shape is classified. In this layer, only one neuron has one state, while all others are cleared. At the same time, it is used to verify the degree of similarity between the shape of the input to the stored patterns and, depending on the evaluation result of the verification, between the response of the upper layer to the lower back. If the returned signal corresponds exactly to the pattern, the classification is completed, and if not, appropriate modifications to the connection weights are made. The individual layers communicate up and down, or resonate by adjusting weights, and the process is repeated until maximum similarity is obtained. The ART network is a learning network in real time, which identifies the shapes that differ significantly from the stored patterns, and, subsequently, memorizes them as new patterns. This network can also correct patterns according to observed deviations. Control and orienting systems monitor these processes (Tadeusiewicz, 1993; Osowski, 2006; Tadeusiewicz et al., 2007).

The most recent models of plant in vitro cultures have been designed using fuzzy hybrid networks. Neurofuzzy logic combines the adaptive learning capabilities of ANNs with the generality of representation from fuzzy logic through simple conditional rules (Shao et al., 2006; Gallego et al., 2011). The usefulness of neurofuzzy logic in effective modeling of complex nonlinear relationships between variables has been proved (Landin et al., 2009; 


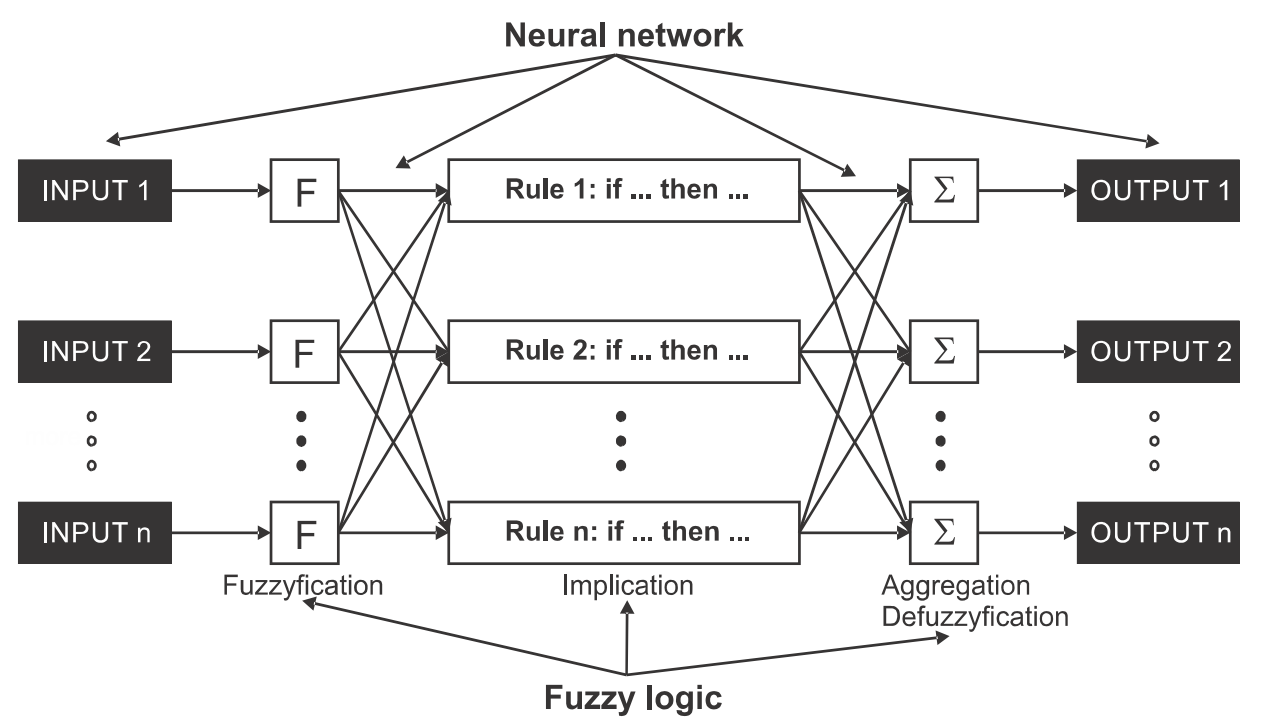

Fig. 5. Structure of a neurofuzzy model

o Area

MLP 3-3-4

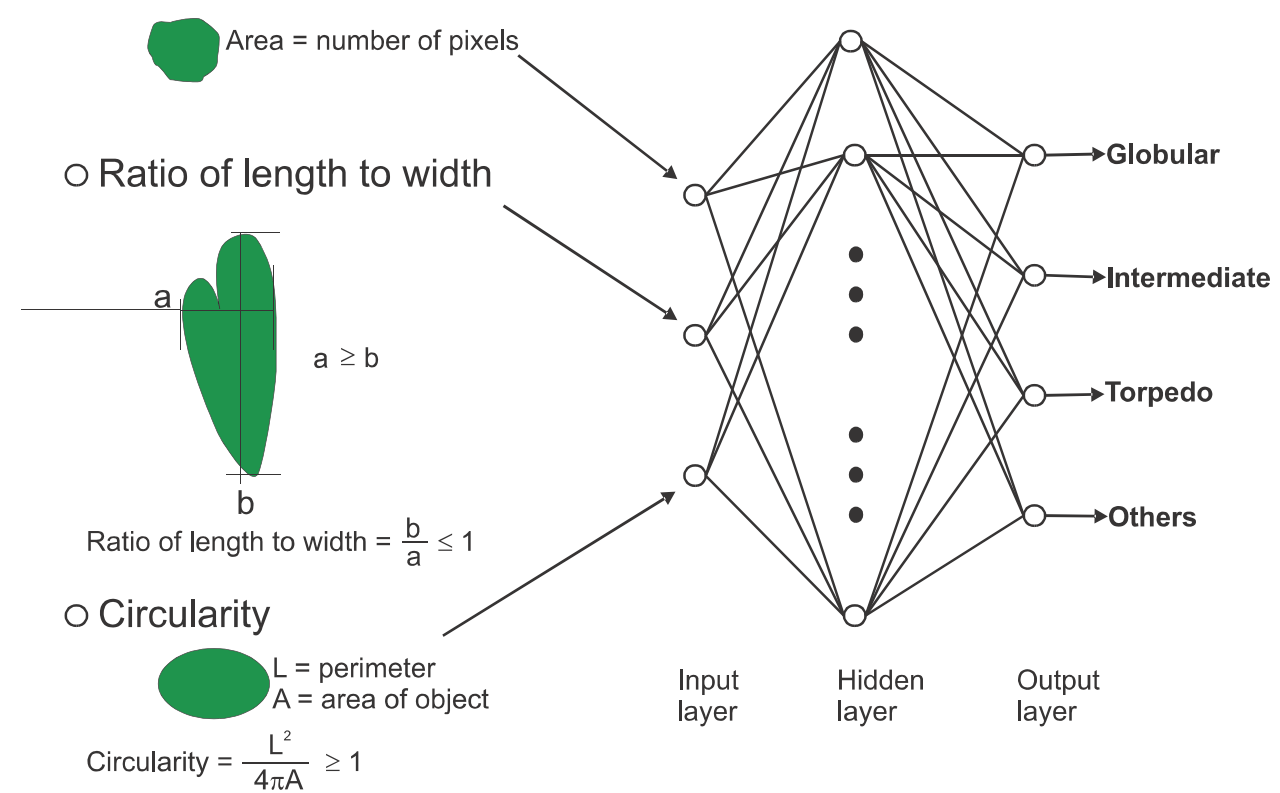

Fig. 6. Structure of ANN for somatic embryos classification (according to Honda et al., 2001)

Gallego et al., 2011). Fuzzy neural network design requires qualitative, but not quantitative, knowledge. Decisions are based on a database of rules. Neurofuzzy logic generates these rules in a clear, understandable format: if there is a prerequisite, then there is a conclusion; thus representing the cause-effect relationships in experimental data. ANNs are used to optimize certain parameters of fuzzy systems in neurofuzzy logic and automatically extract fuzzy rules from numerical data. The simplest approach to designing such systems is to establish rules and membership functions by observing these rules, and then to check how this system operates. If the design of the system does not work, the membership functions can be modified. The network structure used in neurofuzzy logic embeds fuzzyfication, inference and defuzzyfication facilities (Fig. 5). The classic design of the fuzzy model refers to the definition of operations performed at each step: i) fuzzyfication, which involves determining the degree of membership of a particular input to the size of each of the corresponding fuzzy sets covering the 
range of possible input values - this operation is reduced to calculating functions or finding appropriate values in the tables; ii) the use of fuzzy logic operators to determine the extent to which a condition is met in each of the rules; iii) application of the method of implication, which leads to the creation of fuzzy sets corresponding to each of the outputs occurring in the conclusion; iv) aggregation of all output combinations for each of the corresponding output sets and all rules in one fuzzy set; v) defuzzyfication, involving the assignment of a specific value to each of the outputs of the fuzzy set obtained after aggregation. There are numerous implementations of neurofuzzy logic, including algorithms: ANFIS (Adaptive Neuro-Fuzzy Inference Systems), NEFPROX (NeuroFuzzy Systems for Function Approximation) or ASMOD (Adaptive Spline Modeling of Data) (Shao et al., 2006). A commonly used implementation is the ASMOD algorithm, through which the software generates several training models (submodels), and then tests them so as to achieve the best fit to the data. The quality of the models is checked on the basis of various statistical parameters. An ideal model should predict with high probability and define clear and simple conditional rules.

\section{Neural network software}

There are many good software packages that can be used to aid the building neural network models. Neural network software is used to simulate, research, develop and apply ANNs. Generally, the following software is used for the design of ANNs: Statistica Neural Networks, Neural Networks Matlab Toolbox, INForm and FormRules (Intelligensys Ltd, UK), NeuroShell, NeuroSolutions, Alyuda NeuroIntelligence, BioComp iModel, SPSS Neural Connection, etc. However, some of these may be quite expensive. Statistica Neural Networks in the Statistica package is one of the most advanced and best performing ANNs' applications on the market. It has a wide selection of ANN architectures and training algorithms and is easy to use, both for experienced users and for first-time users alike, owing to its automated network search tool. Neural Network Matlab Toolbox can be used in applications such as data fitting, pattern recognition, clustering, time-series prediction, and dynamic system modeling and control (see www. mathworks.com/products/neural-network). INForm is used in modeling and optimizing processes by MLP neu- ral network architecture, standard and accelerated backpropagation algorithms, genetic algorithms and also fuzzy logic (see www.intelligensys.co.uk/inform.htm). FormRules is software for the data mining performed to find models and rules that use neurofuzzy logic (see www. intelligensys.co.uk/formrules.htm). NeuroShell is available as NeuroShell Predictor, NeuroShell Classifier and GeneHunter (genetic algorithm software) and they all are contained in the AI Trilogy package (see www. wardsystems.com). NeuroSolutions is also a powerful and flexible ANN modeling software package, which has an icon-based network design interface with advanced learning procedures and genetic optimization (see www. neurosolutions.com/products/ns). Alyuda NeuroIntelligence supports all stages of ANN design and application (see www.alyuda.com). BioComp iModel is used in nonlinear predictive model development with self-optimizing (see www.biocompsystems.com/products/imodel). SPSS Neural Connection is a powerful combination of ANNs and traditional statistical methods and can be used for classification, prediction, time-series forecasting and clustering. It also includes ANN tools: MLP, RBF, Bayesian neural network and the Kohonen network (see www.spss.com). Gallego et al. (2011) recommended the use of software packages within an easy-to-use interface, i.e. those used in Gago et al. (2010b, d) - INForm and FormRules.

\section{Application of ANNs in plant in vitro cultures}

Neural modeling as a technique is very flexible, effective and versatile in dealing with the nonlinear relationships that prevail in plant tissue cultures. The application of ANNs brings distinct advantages, especially since it does not require knowledge of the nature of the phenomenon under investigation, prior knowledge of the relationship between the variables describing the modelled phenomenon, or knowledge of the distribution of these variables or the relationship between the input and output (Prasad and Dutta Gupta, 2008a). ANNs are, therefore, increasingly used for the interpretation and analysis of data obtained in studies conducted on tissue cultures. Models designed for this purpose are described below and the ANN application in plant in vitro cultures is presented in Fig. 2. This figure shows the structure of the MLP that is usually used for modeling processes in plant in vitro cultures. As can be seen, the data sets 
from in vitro experiments used in individual models are placed in the schematic input layer: i) the $\mathrm{pH}$ value of the medium and its volume and the corresponding weight of the hairy roots from root cultures; ii) the values of $\mathrm{CO}_{2}$, the amount of sucrose and light intensity and corresponding temperatures and growth parameters from micropropagation cultures calculated by the MLP; iii) the Fourier coefficients and corresponding shapes in the output layer and the number of somatic embryos classified by MLP; iv) concentrations of sucrose, glucose and fructose and the corresponding values of biomass in suspension cell cultures; v) digital images of the plants, concentrations of media components and the corresponding number and length of the shoots, also assigned to a given class of plants. Thus, the output layer of the network in Figure 2 provides a summary of all types of obtained results calculated by these models. The publications cited in this paper also describe other types of networks (ART, fuzzy ART, RBF, Kohonen SOM, neurofuzzy logic) used to design models of processes in plant in vitro cultures.

With the use of specialized software, the obtained data can be entered as inputs into the ANN. This software allows selection of the type and architecture of ANN, the transfer function, training algorithm, and model validation, and one can determine its output; and thus, build a model or model the process. However, the final quality of the model depends on the amount of data in the input set.

\section{Classification of somatic embryos}

Somatic embryogenesis, a multi-step in vitro regeneration process, is controlled by a number of different physical and chemical factors and is a promising and economically important way of cloning numerous plant species. Although this process has been used for many valuable crops, there are still several problems, e.g. low germination and conversion rates, which restrict its widespread use (Kępczyńska and Zielińska, 2006, 2011). Somatic embryogenesis is a process whereby somatic cells differentiate into somatic embryos which, in turn, morphologically resemble zygotic embryos. The production of somatic embryos from in vitro cultures and their classification to the appropriate stage of development has been automated with the use of ANNs. A MLP with one hidden layer was applied for the classification of celery somatic embryos and non-embryogenic structures
(Fig. 6). This model also predicted the time needed to transfer the somatic embryos to the next stage of culture (Uozumi et al., 1993; Honda et al., 2001). Four input parameters were used to train the ANN: area, ratio of length to width, circularity, and distance dispersion from images of celery embryos. After training on the basis of the first three parameters, the network was able to classify embryos and non-embryogenic structures, and it also distinguished between globular, heart-shaped and torpedo stages at a level comparable to a human. This ANN was also used to predict the number of plants regenerated from the heart and torpedo embryos after 14 days in the second stage of regeneration.

Classification and pattern recognition models built by ANNs are already being widely used in plant tissue cultures (Prasad and Dutta Gupta, 2008a), usually because the selection of somatic embryos in embryogenic cultures is tedious, costly and time-consuming. A system that recognizes the patterns and morphological features of carrot somatic embryos has been constructed using artificial intelligence technology (Ruan et al., 1997). A hierarchical decision tree consisting of three layers and four nodes was used to obtain an optimal classification. Neural classifiers were incorporated into each node (Fig. 7a). These classifiers grouped the embryos into categories based on the Fourier coefficients, which characterized the morphology of the somatic embryos. These coefficients were obtained in an analysis of the Fourier transform method. In the first node, the embryo population was grouped into four categories: callus, globular stage, torpedoes and "others". The "others" group in the second node was divided into three groups: oblong and heart, secondary, twin and cluster. In the third and fourth node, the ANNs distributed the common groups from the second node into single groups. A three-layer MLP, which was trained by a backpropagation algorithm and constructed in the NeuroShell software, operated in each node. The input layer of all the networks was formed by 34 neurons corresponding to the Fourier properties and the size of the embryos, while the number of neurons in the output layer corresponded to the number of classification groups on that node. The number of neurons in the hidden layer was varied and influenced the network's capacity to solve the problem. Ruan et al. (1997) model categorized carrot somatic embryos with $90 \%$ accuracy or higher. Therefore, the pattern recognition system based on ANNs has 
(A)

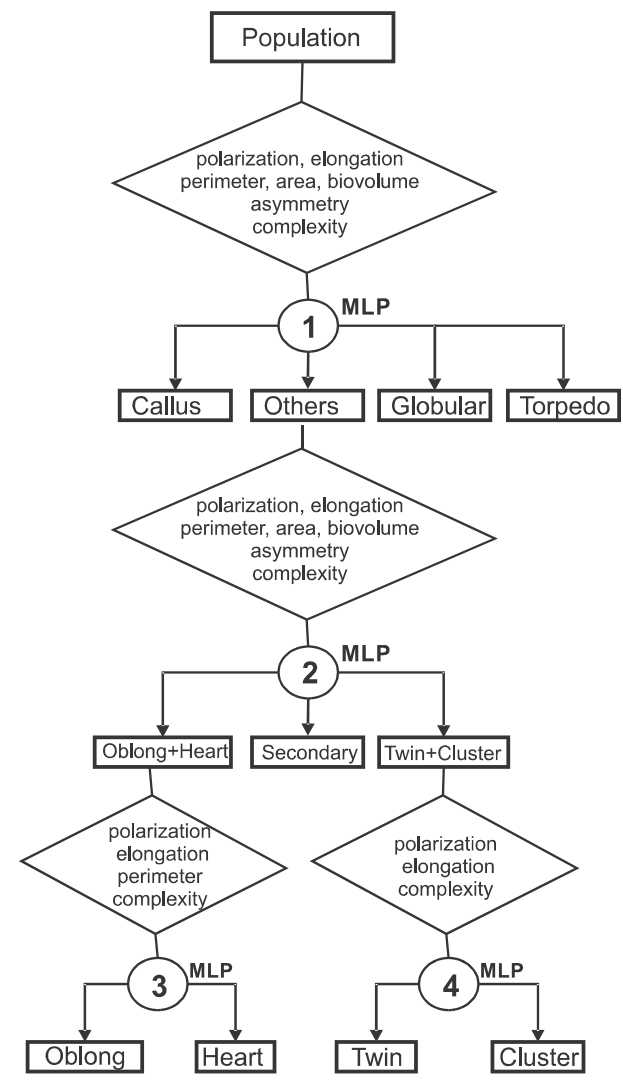

(B)

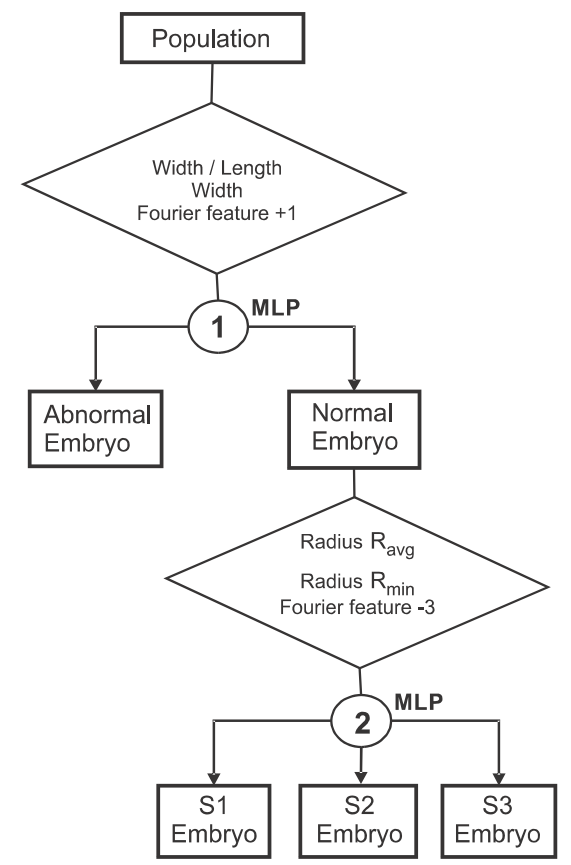

Fig. 7. Hierarchical decision tree with four ANN nodes for somatic embryos classification (A) (according to Ruan et al., 1997) and with two ANN nodes (B) (according to Zhang et al., 1999)

shown great potential for sorting embryos and artificial seed production automation. A system similar to the above classification has applied to the somatic embryos of the Douglas fir (Pseudotsuga menziesii) (Zhang et al., 1999). In this system, the geometric features of somatic embryos were analyzed based on images and were converted to numerical values by a discrete and fast Fourier transform. Then, these values and some of the shape factors were incorporated into an ANN contained in two nodes in a hierarchical decision tree (Fig. $7 \mathrm{~b}$ ). Normal embryos were identified with more than $80 \%$ accuracy. Three-layer MLP topology in NeuroShell software was used to build the ANN. The input layer was composed of 19 neurons representing the radius, length, width, circularity, area, perimeter and the corresponding Fourier coefficients. The hidden layer, which distinguished between normal and abnormal embryos in the first node, consisted of 30 neurons or 25 neurons, when it differentiated the stages of the normal embryos in the second node. The weighting network connections were adjusted via a backpropagation learning algorithm. During the training phase, it was found that Fourier coefficients played a major role in distinguishing between normal and abnormal embryos, and those coefficients that depended on the shape were the main factors for classifying the various stages of development. Zhang et al. (1999) model was evaluated as a useful tool for optimizing the process of the somatic embryogenesis of conifers, and the rejection of abnormal embryos through this model would help to minimize the low frequency of somatic embryo conversion.

\section{Estimation of biomass in plant cell cultures}

The advantage of neural modeling over conventional tools has also been found during the calculation of the mass growth of plant cells (Albiol et al., 1995). In order to estimate the biomass growth of carrot cells (Daucus carota), an MLP was built with bias and one hidden layer (see Table 1). The composition of the hidden layer included three neurons. The input layer consisted of 8 neurons for data on the time of the initial biomass, concentration of sucrose, glucose and fructose. The output 
Table 1. Optimum parameters of ANN model for estimation of biomass in plant cell cultures

\begin{tabular}{c|l|l|c|c|c|c}
\hline $\begin{array}{c}\text { ANN } \\
\text { structure }\end{array}$ & \multicolumn{1}{|c|}{ Inputs } & Outputs & $\begin{array}{c}\text { Number } \\
\text { of hidden } \\
\text { layers }\end{array}$ & $\begin{array}{c}\text { Number } \\
\text { of hidden } \\
\text { neurons }\end{array}$ & $\begin{array}{c}\text { Transfer } \\
\text { function }\end{array}$ & $\begin{array}{c}\text { Learning } \\
\text { algorithm }\end{array}$ \\
\hline MLP 8-3-4 & $\begin{array}{l}\text { time, initial level of inocu- } \\
\text { lum, concentration of glu- } \\
\text { cose, fructose and sucrose }\end{array}$ & $\begin{array}{l}\text { final biomass level, concentra- } \\
\text { tion of glucose, fructose } \\
\text { and sucrose }\end{array}$ & 1 with bias & 3 & sigmoid & $\begin{array}{c}\text { gradient } \\
\text { descent }\end{array}$ \\
\hline
\end{tabular}

layer was composed of four neurons for data on the final biomass levels, sucrose, glucose and fructose. Data for network training were chosen from two different bioreactors with different levels of inoculum and concentrations of sugars. The sigmoid function was selected as the transfer function to designate the output signal for the learning algorithm. The weights were altered, based on the measured mean square error at the output of the network. Then, the gradient descent method in the backward direction was used for the network training. After training by data from two different experiments, the network performance was validated by using a new set of input data. The network approximated better input from two experiments than from one and correctly predicted the changes in sugars and biomass cell suspension. This method successfully measured the levels of sugars and biomass during the cultivation of plant cells. The neural model of Albiol et al. is an efficient alternative to a deterministic mathematical model, even with a small number of experimental data and a minimum amount of information (Prasad and Dutta Gupta, 2008a; Gallego et al., 2011).

\section{Simulation of temperature distribution inside culture vessels}

A controlled microenvironment inside the culture vessels is a critical prerequisite for plant growth in tissue cultures. Environmental factors, such as $\mathrm{CO}_{2}$ concentration, degree of ventilation, light intensity, and air temperature inside the culture vessels, affect the growth of regenerated plants. In particular, an increase in air temperature at high light intensity inhibits growth (Prasad and Dutta Gupta, 2008a). A finite element model (FEM) was constructed using ANN technology to predict the temperature distribution inside the culture vessels. For this model, permanently defined Nusselt numbers $(\mathrm{Nu}$ - heat transfer coefficient) were required for the analysis of heat transfer by forced convection (Suroso et al., 1996; Murase et al., 2008). The ANN was used in this case to calculate the constants as defined by the Nusselt number. Input data for the three-layer MLP were collected from up to four neurons corresponding to the temperatures of nodes defined in the finite element model (Fig. 8). On the basis of the temperature at different air speeds, the ANN permanently estimated the Nusselt equation, and then these coefficients were used to calculate convective heat penetration into the culture vessels. The error between the experimental and simulated temperature was approximately $5 \%$. Due to the randomness of the MLP network input data, all possible combinations of heat transfer coefficients were generated. During network training, the Nusselt equations were directly and accurately determined by the temperature measured during the experiment (Murase et al., 2008). The physical conditions during in vitro culture can be simulated with models such as those described above.

\section{Estimation of shoot length in vitro}

Honda et al. (1997) demonstrated a neural networkassisted estimation of shoot length in in vitro regenerated rice. Data for calculations were taken from digitized images of regenerated cultures. Two different types of ANNs with fuzzy logic (FNN - fuzzy neural network) were used for neural modeling to distinguish between the different regions of the shoot. The FNN-A network was one model with three inputs and outputs, while the FNN-B network consisted of three independent models with three inputs and one output (Fig. 9). Every single network node (neuron) was activated with a sigmoid function. The networks were trained using the backpropagation algorithm. The weights in the trained model were introduced in the form of table rules of colors and compared with one another to obtain the relationships between the colors of the differentiated regions, the 


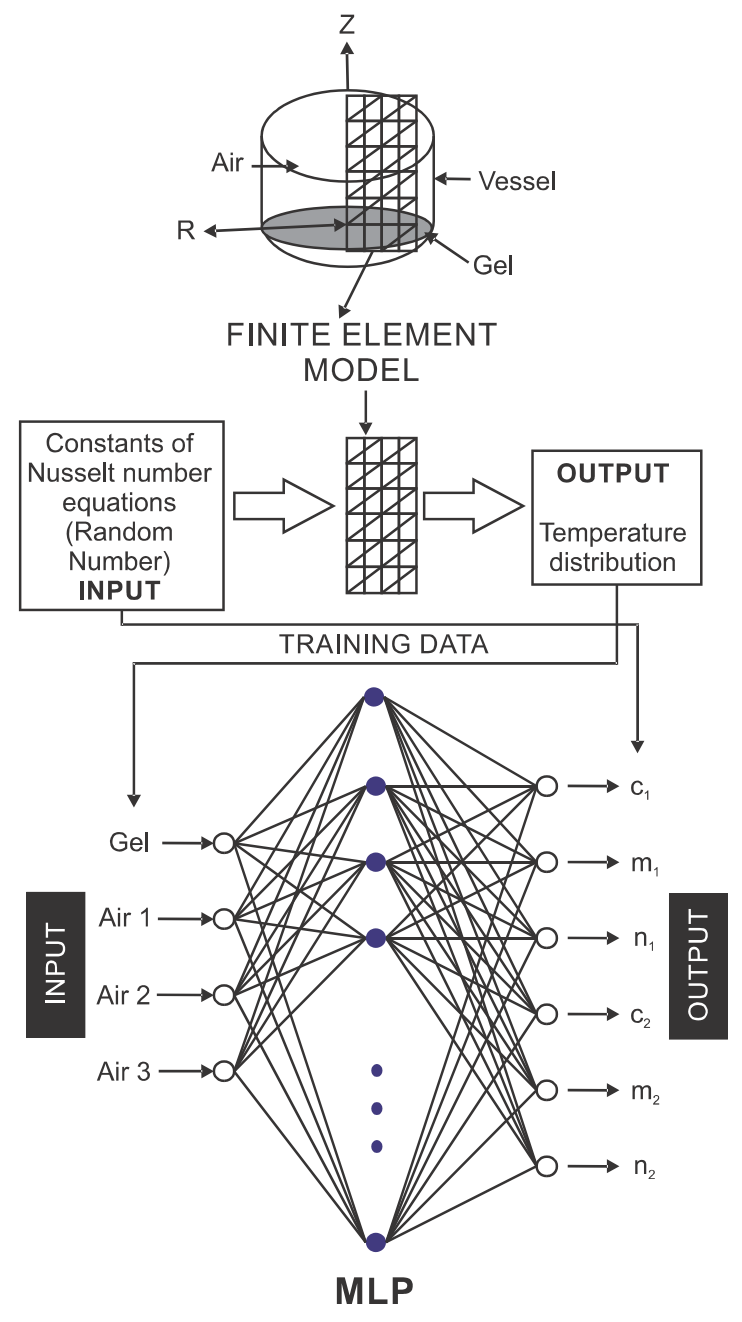

Fig. 8. System with finite element model (FEM) and three-layered MLP neural network for simulation of temperature distribution inside culture vessel (according to Suroso et al., 1996)

callus and the medium. The range of complexity in the mutual relationships between the individual components of the color was computed from the connection weights of the trained network. In this way, the model had a higher degree of accuracy (95\%) in the identification of shoots. The FNN-B model was more effective in identifying the callus regions than the FNN-A. A trinary image was reconstructed based on the outputs of the FNN-B model, which was then subjected to a two-step extraction method of thinning and extraction of the longest path. The shoot region was separated from the rest of the image and its length was calculated. Elongated shoots in the regenerating calluses were measured and compared with values simulated by the network. The mean error between the predicted and experimental shoot lengths was only $1.3 \mathrm{~mm}$ (Honda et al., 1997).
(A)

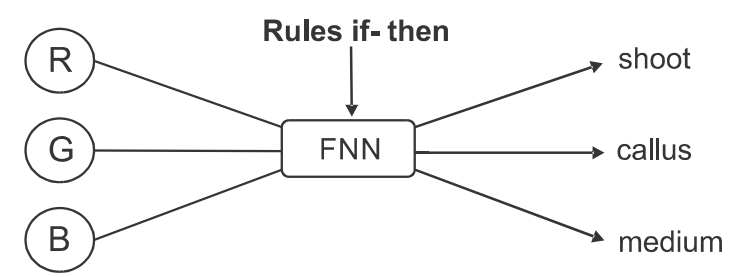

(B)

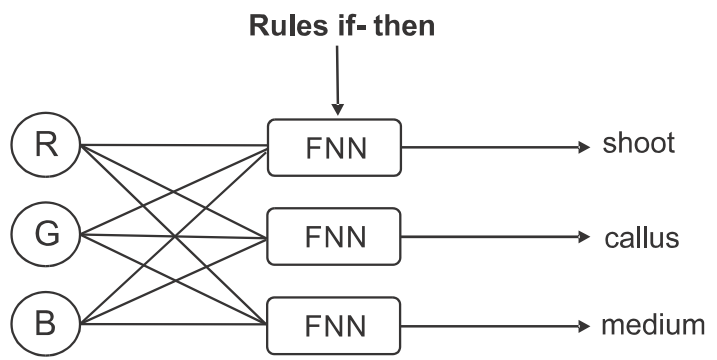

Fig. 9. Structures of FNN-A (A) and FNN-B (B) models for image analysis to estimate shoot length in vitro (according to Honda et al., 1997)

\section{Clustering of in vitro regenerated plants}

Micropropagation through the tissue culture technique is widely used for the propagation of plants. The main obstacle in the commercialization of micropropagation is the low survival rate of in vitro regenerated plants after transfer to ex vitro conditions. Environmental factors in vitro, such as differences in humidity and $\mathrm{CO}_{2}$ concentration or the different distribution of light intensity and air temperature inside the culture vessel, have a particular impact on the quality of the regenerated plants during micropropagation; and thus, result in a diversity of plants (Prasad and Dutta Gupta, 2008a). The development of an automatic decision-making system that reflects the diversity of in vitro regenerated plants was much needed with regard to ensuring the high quality of micropropagation. An ANN was built to assess the quality and to qualify sugarcane plants regenerated in tissue cultures. The ANN was based on photometric parameters which are true indicators evaluating the behavior of cultures capable of regeneration (Honda et al., 1999). The inputs to the ANN were a reflection of the leaf and the intensity of spectral brightness on digitized images.

Similarly, the regenerated plants of gladiolus (Gladiolus hybridus) were sorted by using their photometric behavior: mean brightness, grayscale level, maximum pixel 


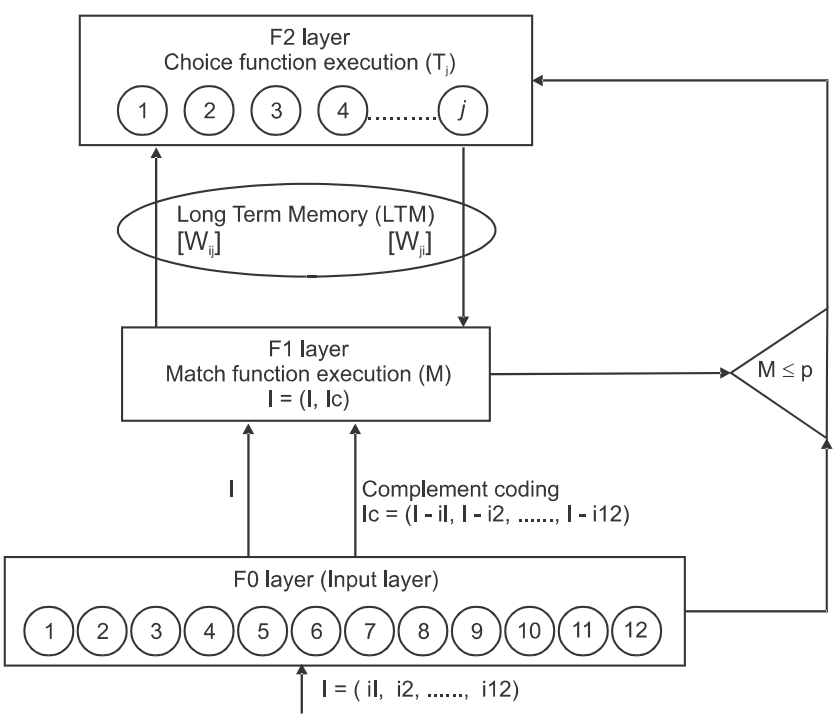

Fig. 10. Fuzzy ART module for clustering in vitro regenerated plants (according to Prasad and Dutta Gupta, 2008b)

count in luminosity and the trichromatic components (red, green and blue (RGB)) from the digitized images of leaves (Mahendra et al., 2004; Prasad and Dutta Gupta, 2008b). Photometric data were the input set for the model constructed using different ANNs: ART2-type resonance (Mahendra et al., 2004; Prasad and Dutta Gupta, 2008b), fuzzy ART (Fig. 10) and Kohonen self-organizing networks (Prasad and Dutta Gupta, 2008b). The ART2 networks and fuzzy ART networks in the models of Mahendra et al. (2004) and Prasad and Dutta Gupta (2008b) were trained using a set of input data from the image extraction of 25 leaves from in vitro regenerated plants, while the validation set consisted of 55 leaves. The ART2-type algorithm grouped the validation set into two classes in a 19:36 ratio, while the fuzzy ART network was separated into seven groups. This separation, however, was wrong; due to the lack of a correlation with the ability of these plants to form corms (Prasad and Dutta Gupta, 2008b). The third type of ANN used for clustering plants, i.e. the Kohonen self-organizing network, is the most common type of network. The self-organizing mapping (SOM) network was designed in Matlab software and has a hexagonal grid topology with a linear function of distance (Fig. 11). A 25-element input set was incorporated into the network. This type is a competitive learning network that is based on input patterns. It produces the output in such a way as to best reproduce dependence in the space of the input vectors. The use of the Kohonen algorithm in the SOM network

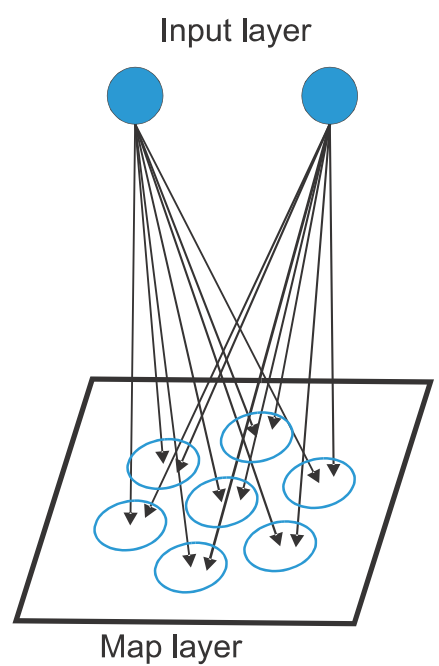

Fig. 11. SOM topology with hexagonal lattice grid (according to Hanafizadeh and Mirzazadeh, 2011)

ordered a validation set of Gladiolus hybridus plants into two groups in a ratio of 28:27 (Prasad and Dutta Gupta, $2008 \mathrm{~b}$ ). Moreover, the biological validation of the regenerated plant groups was determined. It was confirmed which plant groups are more capable of inducing corms. Only in groups differentiated by the ART2 model was there a significant difference: 36.8 and $69.4 \%$ of induced corms, which indicates the potential usefulness of this model.

\section{Predicting optimal conditions for in vitro culture}

Plant tissue culture experiments comprise a part of very complex studies. The growth of plant tissues can be regulated and controlled by changing the composition of the culture media. Optimization of the mineral and plant growth regulator contents is very laborious and time-consuming, and therefore predicting the composition of the growth media and the culture conditions is very useful for choosing the most favorable of these in order to achieve maximum productivity. Neural network software has been successfully used for modeling and optimization of the process of estimating the best in vitro culture conditions (see Table 2). Gago et al. (2010d) developed a neural model to analyze the effect of two variables: sucrose and light on the proliferation of kiwi fruit microshoots (Actinidia deliciosa). For this purpose, commercial INForm v.3.6 software (Intelligensys Ltd, UK) was used. This is an implementation of MLP neural networks with different backpropagation learning algorithms. This software also includes genetic algorithms which serve to 
Table 2. Comparison of ANNs for predicting optimal conditions for in vitro culture

\begin{tabular}{|c|c|c|c|c|c|c|}
\hline $\begin{array}{c}\text { ANN } \\
\text { structure }\end{array}$ & Inputs & Outputs & \begin{tabular}{|c|} 
Number \\
of hidden \\
layers
\end{tabular} & $\begin{array}{c}\text { Number } \\
\text { of hidden } \\
\text { neurons }\end{array}$ & $\begin{array}{l}\text { Transfer } \\
\text { function }\end{array}$ & $\begin{array}{l}\text { Learning } \\
\text { algorithm }\end{array}$ \\
\hline $\begin{array}{l}\text { MLP 2-2-3 } \\
\text { (Gago et al., 2010d) }\end{array}$ & $\begin{array}{l}\text { concentration of sucro- } \\
\text { se, light intensity }\end{array}$ & $\begin{array}{l}\text { response index, } \\
\text { proliferation, } \\
\text { growth }\end{array}$ & 1 & 2 & linear & RPROP \\
\hline $\begin{array}{l}\text { MLP 7-7-1 } \\
\text { (Mehrotra et al., 2008) }\end{array}$ & $\begin{array}{l}\mathrm{pH} \text { and volume of me- } \\
\text { dium, incubation tem- } \\
\text { perature, month of ino- } \\
\text { culation, size, weight } \\
\text { and density of inoculum }\end{array}$ & $\begin{array}{l}\text { weight } \\
\text { of root biomass }\end{array}$ & 1 & 7 & $\begin{array}{l}\text { tansig, } \\
\text { linear }\end{array}$ & $\begin{array}{l}\text { trainrp, } \\
\text { traincgb, } \\
\text { traingda, } \\
\text { traincgf }\end{array}$ \\
\hline $\begin{array}{l}\text { MLP 4-4-1, } \\
\text { RNN 4-4:2-1 } \\
\text { (Prakash et al., 2010) }\end{array}$ & $\begin{array}{l}\text { inoculum density, } \mathrm{pH} \\
\text { and volume of me- } \\
\text { dium, percentage of } \\
\text { sucrose }\end{array}$ & $\begin{array}{l}\text { weight } \\
\text { of root biomass }\end{array}$ & $\begin{array}{l}1 \\
1\end{array}$ & $\begin{array}{c}4 \\
4: 2\end{array}$ & $\begin{array}{l}\text { sigmoid, } \\
\text { radbas, } \\
\text { linear }\end{array}$ & $\begin{array}{l}\text { gradient } \\
\text { descent }\end{array}$ \\
\hline $\begin{array}{l}\text { ANN+HMM 5-3-1 } \\
\text { (Mehrotra et al., 2013) }\end{array}$ & $\begin{array}{l}\mathrm{pH} \text { and volume of me- } \\
\text { dium, concentration } \\
\text { of sucrose and nitrate, } \\
\text { density of inoculum }\end{array}$ & $\begin{array}{l}\text { weight } \\
\text { of root biomass }\end{array}$ & 1 & 3 & $\begin{array}{l}\text { maximum } \\
\text { likelihood }\end{array}$ & Viterbi \\
\hline $\begin{array}{l}\text { Neurofuzzy logic } \\
\text { (Gago et al., 2011) }\end{array}$ & $\begin{array}{l}\text { cultivars of apricot, con- } \\
\text { centration of benzyl- } \\
\text { adenine, } \mathrm{K}^{+}, \mathrm{NO}_{3}^{-}, \mathrm{NH}_{4}^{+} \text {, } \\
\mathrm{Ca}^{2+}, \mathrm{Cl}^{-}, \mathrm{Mg}^{2+}, \mathrm{PO}_{4}^{2-} \\
\mathrm{SO}_{4}^{2-}\end{array}$ & $\begin{array}{l}\text { number of shoots } \\
\text { per explant, } \\
\text { average shoot } \\
\text { length, productivity }\end{array}$ & \multicolumn{2}{|c|}{$\begin{array}{l}\text { submodels } \\
\text { with rules if-then }\end{array}$} & - & $\begin{array}{l}\text { structural } \\
\text { risk } \\
\text { minimisation }\end{array}$ \\
\hline
\end{tabular}

optimize the performance of the models. A detailed description of this software package can be found in Shao et al. (2006). The model of Gago et al. (2010d) was a three-layer MLP with one hidden layer using a linear transfer function and a fast adaptive resilient backpropagation (RPROP) learning algorithm. The input data were concentrations of sucrose in the medium and light intensity. The model outputs were: i) the response index - the mean percentage of explants with at least one microshoot for each combination of sucrose/light, ii) the proliferation - the mean of the number of shoots per explant for each combination and iii) growth - the mean length in $\mathrm{cm}$ of the microshoots for each combination. After training, the network was validated and tested for prediction by using the correlation coefficient R-square $\left(\mathrm{R}^{2}\right)$ parameter. The best in vitro culture conditions were concluded and thus the best combination of factors was estimated for in vitro kiwi fruit proliferation in order to obtain the best results for three parameters: response index, proliferation and growth. Moreover, the neural model for this type of experiment is an alternative to the traditional statistical methods and can be developed, for example, by adding new inputs to the database (the presence of a plant growth regulator) and outputs (survival rate, rooting ability) (Gago et al., 2010d), or also by using another algorithm.

Successful attempts at modeling in vitro culture parameters have also been made in the hairy root cultures of Glycyrrhiza glabra (Mehrotra et al., 2008; Prakash et al., 2010). Initially, the prediction model of in vitro culture conditions was designed along with the inoculum properties for optimum root biomass production (Mehrotra et al., 2008). This model consisted of an MLP performed in Matlab software. The network consisted of a seven-element input layer, one hidden layer consisting of 7 neurons and one output element. The hidden layer had a hyperbolic tangent activation function (tansig) and the output layer included a linear transfer function (purelin). The input data were: $\mathrm{pH}$ and volume of the medium, incubation temperature, the month of inoculation and the inoculum properties: size in $\mathrm{cm}$, fresh weight, and density (number of explants per flask). The output was 
given the total mean fresh weight of root biomass per flask after 40 days of incubation. The best results and those most similar to the experimental data were predicted by the network trained with the trainrp algorithm, but the largest output range was obtained by the network trained with the traincgb algorithm (Mehrotra et al., 2008). Two neural models, also in Matlab (Prakash et al., 2010), were built to evaluate culture parameters such as inoculum density, $\mathrm{pH}$ and medium volume as well as the concentration of sucrose in the culture medium for the hairy roots of Glycyrrhiza glabra. Both models take into account the fact that the biomass fresh weight of roots at the output is a function approximating four input parameters: inoculum density per flask, $\mathrm{pH}$ of growth medium, percentage of sucrose in the growth medium and volume of growth medium per flask. One model was an MLP with the sigmoid activation function (logsig) and a linear transition function in the output layer (purelin) trained with the gradient descent algorithm, while the other was a regression neural network (RNN). All the experimental data from the hairy root cultures were used to test both types of ANNs and were then compared with predicted values (Prakash et al., 2010). Both ANNs were found to be efficient for the prediction of optimal culture conditions to produce large amounts of fresh root weight; yet, the RNN forecasts were more accurate. Subsequently the hybrid model was adapted for hairy root cultures in vitro. This model was based on ANN in combination with a hidden Markov model (HMM) (Mehrotra et al., 2013). The model system for this study was the Agrobacterium rhizogenesmediated hairy root cultures of Rauwolfia serpentina and five test culture conditions as inputs to ANN and HMMs: $\mathrm{pH}$ of liquid growth medium, volume of medium per culture vessel, sucrose and nitrate concentration in the medium and density of initial inoculum per culture vessel and their corresponding fresh weight biomass as the output. Mehrotra et al. (2013) received simulated results for the optimal conditions of maximum fresh weight production from ANN-HMM and pure ANN models. These results were similar to experimental results, but the deviation was lower. The lowest deviation was observed in the simulated results obtained from the combinatorial ANN-HMM model.

Recently, a prediction of the best conditions for in vitro culture has been achieved on data from the micropropagation of apricot (Gago et al., 2011). For this pur- pose, hybrid neurofuzzy logic technology was used which identified the relationships between several factors, such as cultivars, nutrient minerals, plant growth regulators and growth parameters (number and length of shoots and productivity), and extracted biologically useful information from each database to create a model from it. Gago et al. (2011) used FormRules v.3.31 software (Intelligensys Ltd, UK) to create the model. The input data were five varieties of apricots, the concentration of benzyladenine (BA) and different essential mineral elements in the culture media $\left(\mathrm{K}^{+}, \mathrm{NO}_{3}^{-}, \mathrm{NH}_{4}^{+}, \mathrm{Ca}^{2+}, \mathrm{Cl}^{-}, \mathrm{Mg}^{2+}\right.$, $\mathrm{PO}_{4}^{2-}, \mathrm{SO}_{4}^{2-}$ ), while the output growth parameters were the number of shoots per explant, average shoot length and productivity (number of shoots $\times$ average length). Based on this database, the neurofuzzy model presented rules from which it would be possible to obtain the longest apricot shoots. A key factor in controlling shoot growth on the length of the Helena, Lorna and Bulida cultivars was the concentration of the $\mathrm{NH}_{4}^{+}$ions: the average content of $\mathrm{NH}_{4}^{+}$stimulated growth of the shoots. Concentrations of BA had a pronounced effect on shoot length, i.e. the lower the concentration, the longer the shoot, regardless of the $\mathrm{Mg}^{2+}$ concentration. Furthermore, high concentrations of $\mathrm{Ca}^{2+}$ in all of the media promoted the shoot to grow in length. Such a neurofuzzy model quickly answered the question as to which combinations of factors were relevant in obtaining the best results and, at the same time, the model could be applied to different data types (binary, discrete, continuous, images, temporal, fuzzy, historical). In practice, the model generated similar results to those obtained in actual physical experiments, but it was more understandable and usable. Only in the case of one cultivar could the model not predict accurately since the database for this cultivar is not complete. This model can be expanded by including additional information on inputs and outputs, such as additional mineral nutrient concentrations, other growth regulators, organic compounds, physical conditions, etc. (Gago et al., 2011).

\section{Modeling of in vitro rhizogenesis and acclimatization of regenerated plants}

Successful rooting in micropropagation is essential for the in vitro production of plants and can cause problems in some plant species. Acclimatization of these plants to ex vitro conditions may also be limited. Both processes strongly depend on the concentration of auxins (Gago 
et al., 2010a, b). The importance of three relevant factors in rhizogenesis and in the subsequent acclimatization of grapevines (Vitis vinifera L., cv. Mencia and Albariño) was identified using ANN technology (Gago et al., 2010a). In this project, different types of data from plant rhizogenesis and acclimatization phases were integrated. INForm v.3.6 software was used to construct a model for this project; thus, a model of an MLP with the backpropagation learning algorithm was created (see Table 3). Three inputs formed the input layer of the network: cultivar, a concentration of auxin - indole-3-butyric acid (IBA) and IBA exposure time. Then, there was one hidden layer in the network with the asymmetric sigmoid transfer function. The output layer consisted of four outputs: i) the mean number of roots formed from the initial microshoots during rhizogenesis, ii) the mean height of the plants, iii) the mean number of leaves formed ex vitro, and iv) the mean number of nodes of the plants during acclimatization. For modeling, the data set was divided into three groups: training, test and validation data. The backpropagation algorithm RPROP was used to train the model for 2000 epochs. This model was used to predict the optimal combination of input factors in order to obtain the best performance of output parameters during rhizogenesis and then during acclimatization. The model suggested that the IBA exposure time is important only if the concentration of IBA increases in the medium; thus, if the IBA concentration is higher, then the IBA exposure time should be shorter in order to obtain the same number of roots for both grapevine cultivars. Moreover, the Albariño cultivar was more sensitive to the influence of IBA, since it produced more roots than the Mencia cultivar in the presence of IBA in all of the concentrations. This model also indicated that the minimum IBA exposure time or its absence promotes shoot growth per length, regardless of the concentration of IBA. The best results were obtained for the rhizogenesis process in the presence of IBA at the highest concentrations, but a longer exposure time reduced growth during the next phase - acclimatization. In addition, genetic algorithms were used to optimize the model in order to obtain the optimum combination of the IBA concentration and the duration of action for efficient plant production during acclimatization. Thus, the best conditions were inferred from the model, but these were not used during the experiment. These results can be used to optimize experimental conditions in the future. ANN technology allowed the development of a model representing both rhizogenesis and acclimatization which was able to predict different conditions for both processes simultaneously and for two cultivars. It is possible, of course, to develop this model by expanding its database, adding new inputs such as type of media, other plant hormones and new outputs, such as the weight of plants, chlorophyll and carotene content, stomata analysis, etc. (Gago et al., 2010a).

Understanding the cause-effect relationships between culture conditions and plant growth parameters is the basis for the development of high-quality micropropagated plants. Modeling the in vitro rhizogenesis and acclimatization of plants of Vitis vinifera L. cv. Albariño was continued by using the hybrid technology of neurofuzzy logic (see Table 3) (Gago et al., 2010b). A neurofuzzy model was built by using FormRules v.3.31 software. Three variables: auxin type (indole-3-acetic acid (IAA), IBA, $\alpha$-naphthaleneacetic acid (NAA)), the auxin concentration in the medium and the sucrose concentration were incorporated as the inputs, while the number of roots and root length after 28 days of rhizogenesis, survival percentage and plant height after 21 days of acclimatization were the outputs. For each output, a separate submodel was first created and then subjected to training, during which the structural risk minimization method proved to be most accurate. The neurofuzzy model of Gago et al. (2010b) generated four sets of conditional rules for rhizogenesis and acclimatization parameters. Moreover, the optimization of the model was made by selecting a combination so that the best parameters were obtained simultaneously for rhizogenesis and acclimatization. The neurofuzzy model generated on the basis of these results gave a general rule: if the concentration of auxin, IAA, IBA or NAA is medium and the sucrose concentration is medium, then all the parameters of rhizogenesis and acclimatization (number and length of roots, survival and plant height) achieve the highest value (Gago et al., 2010b). Neurofuzzy logic technology has made it possible to answer the question of which combination of factors is optimal to obtain the best (highest) values for plant growth parameters during in vitro rhizogenesis and acclimatization.

\section{Conclusions}

Artificial intelligence technology is currently the most promising and preferred method for modeling com- 
Table 3. Comparison of ANNs for modeling in vitro rhizogenesis and acclimatization of regenerated plants

\begin{tabular}{l|l|l|c|c|c|c}
\hline \multicolumn{1}{c|}{$\begin{array}{c}\text { ANN } \\
\text { structure }\end{array}$} & \multicolumn{1}{|c|}{ Inputs } & Outputs & $\begin{array}{c}\text { Number } \\
\text { of hidden } \\
\text { layers }\end{array}$ & $\begin{array}{c}\text { Number } \\
\text { of hidden } \\
\text { neurons }\end{array}$ & $\begin{array}{c}\text { Transfer } \\
\text { function }\end{array}$ & $\begin{array}{c}\text { Learning } \\
\text { algorithm }\end{array}$ \\
\hline $\begin{array}{l}\text { MLP 3-3-4 } \\
\text { (Gago et al., 2010a) }\end{array}$ & $\begin{array}{l}\text { cultivar, concentra- } \\
\text { tion of IBA, IBA ex- } \\
\text { posure time }\end{array}$ & $\begin{array}{l}\text { mean number of roots } \\
\text { per microshoot, mean } \\
\text { height of the plants, } \\
\text { mean number of leaves } \\
\text { formed } \text { ex vitro, mean } \\
\text { number of nodes }\end{array}$ & 1 & 3 & $\begin{array}{c}\text { asymmetric } \\
\text { sigmoid }\end{array}$ & $\begin{array}{c}\text { RPROP, } \\
\text { genetic } \\
\text { algorithms }\end{array}$ \\
\hline $\begin{array}{l}\text { Neurofuzzy logic } \\
\text { (Gago et al., 2010b) }\end{array}$ & $\begin{array}{l}\text { auxin type, the auxin } \\
\text { concentration, } \\
\text { the sucrose concen- } \\
\text { tration }\end{array}$ & $\begin{array}{l}\text { number of roots, avera- } \\
\text { ge root length, survival } \\
\text { percentage, plant height }\end{array}$ & submodels \\
with rules if-then & - & structural \\
risk \\
minimisation
\end{tabular}

plex biological processes. ANNs can also play a significant role as a prognostic tool with a high potential for modeling studies in plant in vitro cultures. The application of an ANN (Fig. 2) also brings spectacular benefits due to the ANN's ability to capture nonlinear relationships between the data, regardless of their origin or type, and even among incomplete data sets, without the requirement that the user has prior knowledge regarding these data sets. From a practical point of view, neural or neurofuzzy models are capable of predicting what will happen under other circumstances, i.e. those not incorporated into the input to the model. Neural modeling can now be performed with a limited number of experiments, which consequently reduces the costs of plant tissue cultures grown under laboratory conditions and on an industrial scale. Neural modeling serves to aid the development of a virtual workshop that can later be used in a specific way in practice. Finally, adding new inputs and outputs to the model database can easily increase the amount of knowledge derived through the use of ANNs. This may provide a new outlook aimed at understanding the regulatory, developmental and physiological processes in plants. In the future, neural modeling could be used for the mechanization and automation of plant breeding via in vitro cultures and in the segregation of plant tissues in terms of quality, also in aseptic conditions. Moreover, ANNs are very flexible and more useful than other strategies in hybrid models.

\section{References}

Albiol J., Campmajó C., Casas C., Poch M. (1995) Biomass estimation in plant cell cultures: a neural network approach. Biotechnol. Prog. 11: 88-92.
Gago J., Landin M., Gallego P.P. (2010a) Artificial neural networks modeling the in vitro rhizogenesis and acclimatization of Vitis vinifera L. J. Plant Physiol. 167: 1226-1231.

Gago J., Landin M., Gallego P.P. (2010b) A neurofuzzy logic approach for modeling plant processes: $A$ practical case of in vitro direct rooting and acclimatization of Vitis vinifera L. Plant Sci. 179: 241-249.

Gago J., Landin M., Gallego P.P. (2010c) Strengths of artificial neural networks in modelling complex plant processes. Plant Signal. Behav. 5(6): 743-745.

Gago J., Martinez-Núńez L., Landin M., Gallego P.P. (2010d) Artificial neural networks as an alternative to the traditional statistical methodology in plant research. J. Plant Physiol. 167: 23-27.

Gago J., Pérez-Tornero O., Landin M., Burgos L., Gallego P.P. (2011) Improving knowledge of plant tissue culture and media formulation by neurofuzzy logic: A practical case of data mining using apricot databases. J. Plant Physiol. 168: 1858-1865.

Gallego P.P., Gago J., Landin M. (2011) Artificial neural networks technology to model and predict plant biology process. In: Artificial neural networks - methodological advances and biomedical applications, ed. Suzuki K., InTech, Rijeka: 197-216.

Hanafizadeh P., Mirzazadeh M. (2011) Visualizing market segmentation using self-organizing maps and Fuzzy Delphi method - ADSL market of a telecommunication company. Expert Syst. Appl. 38: 198-205.

Honda H., Ito T., Yamada J., Hanai T., Matsuoka M., Kobayashi T. (1999) Selection of embryogenic sugarcane callus by image analysis. J. Biosci. Bioeng. 87: 700-702.

Honda H., Liu C., Kobayashi T. (2001) Selection of embryos. In: Advances in Biochemical Engineering/Biotechnology, ed. Sheper T., vol. 72. Springer-Verlag, Berlin, Heidelberg: $177-178$.

Honda H., Takikawa N., Noguchi H., Hanai T., Kobayashi T. (1997) Image analysis associated with a fuzzy neural network and estimation of shoot length of regenerated rice callus. J. Ferment. Bioeng. 84: 342-347. 
Kepczyńska E., Zielińska S. (2006) Regulation of Medicago sativa L. somatic embryos regeneration by gibberellin $A_{3}$ and abscisic acid in relation to starch content and $\alpha$ amylase activity. Plant Growth Regul. 49: 209-217.

Kępczyńska E., Zielińska S. (2011) Disturbance of ethylene biosynthesis and perception during somatic embryogenesis in Medicago sativa L. reduces embryos' ability to regenerate. Acta Physiol. Plant. 33: 1969-1980.

Kosiński R. (2002) Artificial neural networks, nonlinear dynamics and chaos. WNT, Warsaw, (in Polish).

Landin M., Rowe R.C., York P. (2009) Advantages of neurofuzzy logic against conventional experimental design and statistical analysis in studying and developing direct compression formulations. Eur. J. Pharm. Sci. 38: 325-331.

Mahendra, Prasad V.S.S., Dutta Gupta S. (2004) Trichromatic sorting of in vitro regenerated plants of gladiolus using adaptive resonance theory. Curr. Sci. 87: 348-353.

Mehrotra S., Prakash O., Mishra B.N., Dwevedi B. (2008) Efficiency of neural networks for prediction of in vitro culture conditions and inoculum properties for optimum productivity. Plant Cell Tiss. Organ Cult. 95: 29-35.

Mehrotra S., Prakash O., Khan F., Kukreja A.K. (2013) Efficiency of neural network-based combinatorial model predicting optimal culture conditions for maximum biomass yield in hairy root cultures. Plant Cell Rep. 32: 309-317.

Murase H., Okayama T., Suroso (2008) Intelligence inverse analysis for temperature distribution in a plant culture vessel. In: Plant Tissue Culture Engineering, eds. Dutta Gupta S., Ibaraki Y. Focus on Biotechnology, series eds. Hofman M., Anné J., vol. 6. Springer-Verlag, Berlin: 373394.

Osowski S. (2006) Neural networks for information processing. Warsaw University of Technology Publishing House (OWPW), Warsaw, (in Polish).

Prakash O., Mehrotra S., Krishna A., Mishra B.N. (2010) $A$ neural network approach for the prediction of in vitro culture parameters for maximum biomass yields in hairy root cultures. J. Theoretical Biol. 265: 579-585.

Prasad V.S.S., Dutta Gupta S. (2008a) Applications and potentials of artificial neural networks in plant tissue culture.
In: Plant Tissue Culture Engineering, eds. Dutta Gupta S., Ibaraki Y. Focus on Biotechnology, series eds. Hofman M., Anné J., vol. 6. Springer-Verlag, Berlin: 47-67.

Prasad V.S.S., Dutta Gupta S. (2008b) Photometric clustering of regenerated plants of gladiolus by neural networks and its biological validation. Comput. Electron. Agr. 60: 8-17.

Ruan R., Xu J., Zhang C., Chi C.-M., Hu W.-S. (1997) Classification of plant somatic embryos by using neural network classifiers. Biotechnol. Prog. 13: 741-746.

Sadat Noori S.A., Ebrahimi M., Khazaei J., Khalaj H. (2011) Predicting yield of wheat genotypes at different salinity by artificial neural network. Afric. J. Agric Res. 6: 2660-2675.

Shao Q., Rowe R.C., York P. (2006) Comparison of neurofuzzy logic and neural networks in modelling experimental data of an immediate release tablet formulation. Eur. J. Pharm. Sci. 28: 394-404.

Sherrod P.H. (2013) DTREG - Predictive modeling softwaremanual, http://www.dtreg.com/DTREG.pdf.

Suroso, Murase H., Tani A., Honami N., Takigawa H., Nishiura Y. (1996) Inverse technique for analysis of convective heat transfer over the surface of plant culture vessel. Trans ASAE 39: 2277-2282.

Tadeusiewicz R. (1993) Neural Networks. Academic Publishing House, Warsaw, (in Polish).

Tadeusiewicz R., Gąciarz T., Borowik B., Leper B. (2007) Exploring the properties of neural networks using programs in $C$. Publisher Polish Academy of Sciences, Krakow, (in Polish).

Uozumi N., Yoshino T., Shiotani S., Suehara K., Arai F., Fukuda T., Kobayashi T. (1993) Application of image analysis with neural network for plant somatic embryo culture. J. Ferment. Bioeng. 76: 505-509.

Zhang C., Timmis R., Hu W.-S. (1999) A neural network based pattern recognition system for somatic embryos of Douglas fir. Plant Cell Tiss. Organ Cult. 56: 25-35.

Zhang G.P. (2008) Neural networks for data mining. In: Soft computing for knowledge discovery and data mining, eds. Maimon O., Rokach L., Springer, New York: 17-44. 